\title{
Student perspectives on mental health support and services in the UK
}

Rachel Batchelor ${ }^{1}$, Emma Pitman $^{1}$, Alex Sharpington ${ }^{1}$, Melissa Stock $^{1}$ and Eilidh Cage ${ }^{1}$

${ }^{1}$ Royal Holloway, University of London, Surrey, UK

Corresponding author: Dr Eilidh Cage, Department of Psychology, Royal Holloway,

University of London, Egham, Surrey, UK. Email: eilidh.cage@ @rhul.ac.uk, Tel: (+44)1784 276523.

Manuscript accepted for publication in Journal of Further and Higher Education on 3/2/19

This is an Accepted Manuscript of an article published by Taylor \& Francis

Group in Journal of Further and Higher Education on 1 Mar 2019, available online: http://www.tandfonline.com/10.1080/0309877x.2019.1579896 


\begin{abstract}
Student mental health is an issue of growing concern. Past research indicates that many students are not accessing the support they require, which may be in part due to issues surrounding the standards of the available services. Using a participatory framework, the current study utilised a mixed methods design to examine student experiences of NHS mental health services and perspectives of peer support. 376 UK students completed an online survey examining their experiences of NHS mental health services, as well as their attitudes towards peer support. Several improvements were identified for future NHS mental health services regarding reduced waiting times, better access to alternative treatments and facilitating more patient-centred communication. Benefits of peer support services were also noted, including the potential to normalise experiences and promote belonging. These findings have implications for understanding how we can best support students experiencing mental health difficulties.
\end{abstract}

Key words: student mental health; student wellbeing; support services; National Health Service; peer support 
The topic of student mental health is one of great concern for universities and mental health services alike. In 2016/17, 53,045 students had a recorded mental health condition (Higher Education Statistics Authority 2018), with the number disclosing mental health conditions increasing over the past ten years (Thorley 2017). However, it is likely that mental health difficulties are more prevalent than recorded statistics, with under half of students with a mental health condition estimated to not report this to their university (Thorley 2017). Mental health difficulties impact on students' ability to achieve their full academic potential (Eisenberg, Golberstein and Hunt 2009) and those with mental health difficulties are less likely to complete their studies (Thorley 2017). It is therefore vital that students' experiences of mental health services are understood, as well the support students wish to receive, to ensure their needs are being met.

Research indicates that students are not receiving the support they require, with few able to access support within a week (NUS-USI 2017) and around three quarters of students with mental distress not receiving counselling (Rosenthal and Wilson 2008). In a study of US universities, Mowbray et al. (2006) outlined key roles of university counsellors and campusbased mental health services for supporting students with mental health difficulties, making suggestions for improving these services, such as ensuring accessible appointments and welltrained staff. In the UK, university counselling has been noted as beneficial for both internalising (e.g. emotional responses directed inwards onto the self) and externalising (e.g. emotional responses directed outwards (Bask 2015)) problems (Biasi et al. 2017). However, many students report being unaware of the location or how to access their Counselling or Disability Services (Gulliver et al. 2018). In Quinn et al.'s (2009) qualitative study, students reported a reluctance to seek help from such services due to concerns about stigma. These 
findings suggest that there are a number of issues with students being able to access appropriate mental health services.

In the UK, provision of free healthcare via the National Health Service (NHS) may make the experiences of UK students unique. Between 2011 and 2016, 94\% of university institutions reported an increase in students accessing support services, including those provided by the NHS (Thorley 2017). Among the general population, problems have been identified with NHS mental health services, including long waiting lists - 54\% of patients report waiting over three months to start treatment, with $12 \%$ waiting for over a year (Docherty and Thornicroft 2015). There are also reports of poor access to referrals and issues with communication between services (Docherty and Thornicroft 2015; Kendall et al. 2012; Newman et al. 2015). Funding is also a significant issue, with a $£ 260$ million shortfall in funding occurring since 2005 , leading to $40 \%$ of mental health trusts having below acceptable levels of staffing (Docherty and Thornicroft 2015). Students report benefiting from a link between NHS and university support services (Gulliver et al. 2018). However, there is little research examining students' experiences of accessing NHS support. Understanding the experiences of students could act as a valuable resource to ensure the needs of future students are met, as the current study intends to examine.

Despite the need for support, many UK campuses do not provide students with access to NHS mental health specialists capable of delivering treatment on campus; with a quarter not working closely with NHS secondary mental health services at all (Thorley 2017). When students do access support, they report mixed, often negative, experiences of using their General Practitioner (GP) for mental health issues, with GPs often recommending medication rather than other forms of support (Quinn et al. 2009). As such, current standards of support may not be adequate to deal with the problem of student mental health, but research is needed 
to confirm that this is the case. Therefore, the current study aimed to examine UK students' experiences of accessing NHS mental health support services.

Another aim of this study was to examine students' attitudes towards alternative types of support for mental health issues, specifically peer support. Peer support is offered by others with lived experience of similar mental health problems (Shaw 2014). Potential benefits of peer support include increased wellbeing and self-esteem, a sense of hope, companionship and improved social functioning (Davison et al. 2012; Lawton-Smith 2013). Through semistructured interviews with students, McBeath, Maureen and Bohn (2017) reported that peer support could increase sense of belonging within the university community. Through sharing experiences, peer support may also provide greater social engagement and group identification (Naslund et al. 2014). Sense of group identification has been shown to predict lower levels of depression (Cruwys et al. 2006), higher levels of life satisfaction (Sani et al. 2012) and well-being (McBeath et al. 2017).

Students with poor mental wellbeing and those who have accessed professional support before are the most likely to use peer support (Byrom 2018). Byrom's (2018) found that most participants returned for multiple sessions of a peer-led course for mild depression, with a third completing all available sessions and those with lower levels of mental wellbeing more likely to complete the course. Three-quarters of participants reported that they were more confident talking about their mental health post-intervention, noting the sessions helped them improve their ability to take care of their own mental health (Byrom 2018). Further, Cunningham et al. (2017) found that when students picked between hypothetical mental health services, $40 \%$ reported that they would prefer to talk to peers who had similar experiences over professional services. Peer support may thus offer a useful and desired alternative to professional services. 
However, the lack of available peer support, including the limited periods of availability, has been criticised, with participants wanting more support schemes, including more user-led peer support (Shaw 2014). Despite the potential benefits of peer support, there is a paucity of research into the desire for peer support within university students and their knowledge of peer support, a gap in the literature which the current study aimed to fill. Using both qualitative and quantitative methods, this study examined UK students' views of peer support, in terms of the potential benefits and barriers of peer support.

As discussed, students report several issues with the current standards of mental health services at university. Highlighting the ways in which students themselves believe the problems could be overcome could lead to positive changes that benefit students. Ultimately, this could lead to crucial changes in mental health procedures across universities and allow for better access to support for students with mental health difficulties. As such, the current study used mixed methods to examine students' experiences of accessing support for their mental health via the NHS, and their views of the potential benefits and challenges of peer support. This study utilises an inductive and semantic approach, aiming to identify surfacelevel themes derived from the data (Braun and Clarke 2006). 


\section{Methods}

\section{Participants}

Participants included 376 students studying in the UK. There were 314 females $(83.5 \%), 59$ males $(15.7 \%)$ and 3 people who reported 'other' genders or preferred not to say $(.8 \%)$. The mean age was $20.73(S D=3.34)$ and $76.9 \%$ of the sample was White British. $12 \%$ reported other White ethnicities, 5.6\% were Asian/British Asian, 3.7\% were of mixed-race and .5\% were Black British/African/Caribbean. Most of the sample identified as heterosexual (76.8\%), with $12.6 \%$ identifying as bisexual and $7.7 \%$ as homosexual. Participant recruitment took place via social media sites, such as Facebook and Instagram, as well as through word of mouth and the 'Student Minds' website.

In terms of university demographics, most participants were studying full-time (97.9\%) in universities in the South East of England (65.3\%), with other students coming from across the UK, including Wales (6.8\%) and Scotland (1.2\%). Most were home students (86.2\%), with 9\% EU and 4.8\% international students. Most were studying for their undergraduate degree (89.1\%), with $6.6 \%$ studying their Masters and $3.7 \%$ a PhD. $26.6 \%$ of participants were in their first year of study, $28.5 \%$ in their second, $39.6 \%$ in their third and $5.1 \%$ in their fourth year of study. The majority lived away from home $(86.7 \%)$ rather than commuting to university $(13.3 \%)$.

Ethical approval was obtained via Royal Holloway, University of London and all participants gave full informed consent before participating.

\section{Materials}


Participants completed an online survey presented using the 'Qualtrics' survey software. After giving informed consent, participants were asked to self-report any diagnosed mental health conditions. To characterise the sample, details were recorded on specific diagnoses (e.g. anxiety disorder, mood disorder), the timing of their diagnosis (before or during university) and age at diagnosis. Further, participants reported suspected mental health conditions and whether they were currently seeking diagnosis.

Experiences of NHS mental health services.

Opinions on NHS mental health services were measured using questions adapted from the Service User Questionnaire (Commission for Healthcare Audit and Inspection 2008). Participants were first asked which services they had accessed with regards to their mental health since they had been at university (e.g. GP, counselling or Community Psychiatric Nurse) and where they had access to their GP (e.g. within university, near university or near hometown). They were then asked if they had seen a GP in the last 12 months to specifically discuss their mental health. If they answered yes, they rated qualities of their GP in terms of feeling listened to and treated with respect, if they had trust/confidence in their GP, and if they had had enough time to discuss their mental health. Participants could respond with 'yes, definitely', 'yes, to some extent' or 'no'.

All participants were asked how long they been in contact with the NHS mental health services (from 'never' to 'more than ten years ago'). If participants had been in contact with NHS mental health services, they were asked when they last saw someone from NHS mental health services (from 'in the last week' to 'more than six months ago'). These participants were also asked if they had received any counselling sessions (e.g. talking therapies) from the NHS in the last 12 months and whether they had wanted talking therapies during this time. If 
participants had received talking therapies, they were asked if they had found it helpful ('yes, definitely', 'yes, to some extent' or 'no').

Participants who had accessed NHS mental health services rated the care they had received in the last 12 months on a 5-point Likert scale ('very good' (1) to 'very poor' (5)). They were asked whether they had enough say in decisions about their care and treatment in relation to their mental health and whether their mental health diagnosis had been discussed with them ('yes, definitely', 'yes, to some extent' or 'no'). Finally, those who had been in contact with NHS mental health services were asked two open-ended qualitative questions: "Is there anything particularly good about your care?" and "Is there anything that could be improved?"

\section{Peer Support.}

Peer support was investigated using questions adapted from the Peer Support in Secure Services Report (Shaw 2014). Participants selected the peer support services (e.g. drop-in sessions, activity or condition-based groups, one-to-one support) which were available within their university and which support they believed to be required from the same list. They were then asked whether they would access peer support if it were available ('yes', 'no' or 'not sure'). Next, they selected their main reason for accessing peer support (e.g. 'meeting new people \& making friends', 'because you are interested in the activities'). Participants also indicated their preferred time of day to access peer support.

Participants then selected possible benefits of peer support over staff support from a list of choices, such as 'greater choice', 'fewer restrictions' and 'greater insight and understanding'. Next, they indicated whether they thought peer support would be beneficial for their wellbeing ('yes' or 'no'). This question was followed by an open textbox where participants could provide further details to explain their preference. Participants were asked whether they thought more user-led peer support (support in which peers set the agenda, rather than staff) 
could occur at their university. Finally, participants were asked to select the reasons that would prevent them from accessing peer support (e.g. stigma, lack of interest).

\section{Procedure}

This study used a participatory research methodology (Cornwall and Jewkes 1995), where students themselves were placed as the researchers. Four undergraduate, final year Psychology students worked with an academic member of staff in a Psychology Department to develop the project, drawing on lived and peer experience to identify the research aims.

In the survey, participants first completed demographic information (about themselves and their studies) and information about their mental health conditions. Participants were asked whether they had received support for their mental health during their time at university. They then completed questions regarding experiences of NHS services, followed by peer support questions. The survey took approximately 30 minutes (as part of a broader study not reported in full here) and upon completion, participants were debriefed and signposted to resources. Data was collected between December 2017 and February 2018.

To analyse qualitative responses, conventional content analysis was used. This was deemed the most appropriate form of qualitative analysis due to the large number of responses, and the need to find responses with the highest relevance. Two independent raters read all responses and created categories before meeting to discuss and agree categories. Responses were then independently recoded into these categories, any disagreements were discussed, and final coding agreed. Following the guidelines for agreement by Landis and Koch (1977) inter-rater reliability was assessed using Cohen's kappa coefficients and is reported within the results section.

\section{Design}


A mixed-methods cross-sectional survey was used to examine student's experiences of NHS mental health services and opinions of peer support. Quantitative data was collected on students' use of and attitudes towards NHS mental health care and peer support.

Conventional content analysis was used to examine students' opinions on the positives and negatives of current NHS mental health care, and why peer support would be beneficial or disadvantageous. The conventional approach is inductive in nature, aiming to identify categories that 'flow from the data' (Hsieh and Shannon 2005). 


\section{Results}

\section{Mental health conditions.}

151 participants $(40.2 \%)$ reported a diagnosed mental health condition. Most often, participants reported an anxiety disorder $(30.3 \%, n=114)$ or $\operatorname{mood}$ disorder $(22.3 \%, n=84)$. Table 1 shows all diagnoses as well as the percentage who had received their diagnosis while at university and median age of diagnosis - indicating that most participants had been diagnosed prior to university. $44.1 \%(n=166)$ suspected an undiagnosed mental health condition. Of these participants, $17.5 \%(n=29)$ reported that they were seeking a diagnosis and $82.5 \%(n=137)$ said they were not seeking a diagnosis. Most participants suspected an anxiety disorder $(25.5 \%)$ or a mood disorder $(17 \%)$.

[Insert Table 1 here]

\section{Accessing support.}

Since being at university, over half of the sample $(55.9 \%, n=210)$ had accessed support for their mental health. The most commonly reported form of support came from GP consultations (40.4\%), followed by university counselling services $(31.6 \%)$ and university mental health advisers (20.5\%; Table 2).

\section{[Insert Table 2 here]}

In terms of where participants accessed their GP, $48.7 \%$ used GP services within their university, $38.8 \%$ used GP services near their home and $12.6 \%$ used a service near their university. In the last 12 months, $39.1 \%$ had seen a GP to discuss their mental health. Those participants who had seen a GP in the last 12 months rated the qualities of their GP (Table 3). Participants reported that they did not have adequate time to discuss their condition or 
treatment, but the majority felt they had been treated with respect, trusted their GP and had been listened to.

[Insert Table 3 here]

\section{Experiences of NHS mental health services.}

In terms of contact with NHS mental health services, $12 \%$ had been in contact for one year or less, $13.8 \%$ for one to five years, $5.6 \%$ for six to ten years, and $1.9 \%$ for more than ten years. $5.9 \%$ could not remember or preferred not to say. $60.6 \%$ reported that they had never been in contact with these services. Of the participants who stated that they been in contact with NHS mental health services, $14.5 \%$ had been in contact within the last week, $10.5 \%$ within the last month, $25 \%$ within the last one to three months, $7.3 \%$ within the last four to six months and $42.7 \%$ had been in contact more than six months ago.

For those that had been in contact with NHS mental health services, $44 \%$ had attended talking therapies (e.g. counselling) within the last 12 months and 53.6\% said that they wanted talking therapies. Of those who had received talking therapy, $41 \%$ reported that it was not helpful, $45.9 \%$ that it was helpful 'to some extent' and $13.1 \%$ rated it as definitely helpful. Those who had received care from NHS mental health services rated the quality of their care over the last 12 months $-34.2 \%$ rated it as either 'very good' or 'good'; $30.8 \%$ rated it as 'fair' and $35 \%$ rated it as either 'poor' or 'very poor'. These participants also reported whether they felt they had enough say in the decisions about their treatment and care $-22.1 \%$ said 'Yes, definitely', 47.5\% 'Yes, to some extent', and 30.3\% said 'No'. When asked if their mental health diagnosis had been discussed with them, 18.5\% said 'Yes, definitely', 48.4\% 'Yes, to some extent' and $33.1 \%$ said 'No'.

Content analysis. 
Of 125 participants who had been in contact with NHS mental health services, 40 (32\%) provided qualitative responses when asked if there had been anything particularly good about their care. Four broad categories were identified and agreed: NHS staff qualities, patientcentred communication and care, access to services and the advice provided. The most common response was positive comments on NHS staff qualities $(n=25)$, with participants indicating that many staff were patient and supportive: "Everyone I have spoken to has been very patient and kind which I really appreciate". The second most common response $(n=13)$ was that care had been patient-centred, with a clear focus on what the individual wanted for their mental health needs: "It was very much driven by myself identifying goals that I wanted to achieve". Some participants $(n=6)$ also mentioned access to services: they had found it easy to get an appointment and they were seen regularly within the NHS: "I was seen to really quickly and put into counselling really quickly". Three participants mentioned that they had been satisfied with the advice provided, specifically regarding managing their symptoms and seeking appropriate services: "It was the psychiatrist who suggested that I was autistic and encouraged me to seek a diagnosis". For inter-rater reliability, three categories had almost perfect agreement (.81 - .91) and one category had substantial agreement (.70).

Of 125 participants who had been in contact with NHS mental health services, 73 (58\%) provided responses when asked if there was anything that could be improved about these services. Content analysis identified seven categories: waiting times, access, patient-centred, NHS staff, more effective treatments, multidisciplinary care, and follow-up care. Three subcategories were identified within 'access', and two subcategories were identified within 'NHS staff' and 'patient-centred' respectively (Figure 1).

[Insert Figure 1 here] 
The most reported category was waiting times $(n=26)$, with participants indicating that the time spent waiting to be seen by NHS mental health services needed to be reduced: "After a suicide attempt it took me 6 months to get an NHS psychiatrist appointment"; "IAPT in my area [has a] 18-24 months waiting time". The second most reported category was access, specifically access to alternative treatments $(n=20)$ : “Offering additional or different treatment if it's clear that one treatment isn't working"; more regular/longer appointments ( $n$ = 8): "More frequent sessions would really benefit me") and referrals $(n=7)$ : "I need help but I can't [get] it because I'm not sick enough yet, they have left me in a relapse, and until I reach crisis point no one will intervene".

The third most reported category was patient-centred aspects, specifically patient-centred communication $(n=16)$ and patient-centred care $(n=13)$ - participants expressed a desire to be listened to more during discussions with NHS staff: "When I spoke with my doctor he had the page for over-the-phone therapies up before I even sat down, the outcome of the meeting was going to be the same regardless of what I had to say". Some participants also commented that staff qualities needed to be improved, such as being more compassionate and caring $(n=$ 13): "They didn't seem to care, they just wanted to move onto the next person" and knowledgeable $(n=8)$ "Making all doctors aware of how mental health can affect people and ensure people aren’t sent away”.

Some participants also commented on the need for more effective treatments $(n=12)$, with participants expressing their concerns with current services: "I have had an initial session in both counselling and CBT and neither have given me much optimism or hope”. Some participants identified concerns over multidisciplinary care $(n=7)$, such as lack of communication between services - especially when being discharged from one service and passed on to another: "There needs to be more coordination between university-owned 
counselling services, GPs, and community mental health support”. Finally, some participants reported a desire for more follow-up care $(n=6)$, with participants' reporting a lack of continued support during the periods of no therapy: "Attentiveness and follow ups on how I feel [is needed]". Inter-rater reliability for seven categories was almost perfect agreement (.81- .97) and four categories had substantial agreement $(.61-.80)$.

\section{Peer support.}

Participants were asked about the types of peer support available to them at their university, followed by the types of support they thought were needed (Table 4). Most often, participants reported that activity groups were available (53.2\%), followed by relaxation and mindfulness (41.5\%) and telephone/online support (41\%). In terms of the types of peer support that participants thought were needed, one-to-one support was the most desired type (56.6\%), followed by support in a crisis $(54.5 \%)$. The greatest discrepancy between the support available and the support desired was for support in a crisis.

\section{[Insert Table 4 here]}

When asked about use of peer support, $48.1 \%$ reported that they were unsure if they would access peer support, while $34 \%$ said they would and $17.8 \%$ would not. If they were to access peer support, they preferred to access it Monday to Friday between 5pm - 9pm (41\%) or had no preference of timings (23.9\%). In terms of reasons why they would access peer support, the majority selected that it was 'something to do' (94.3\%), while 37\% would access it to support their recovery, $25 \%$ would use it to meet new people and $10.9 \%$ would be interested in the activities.

In terms of benefits, $77.4 \%$ thought that peer support would be beneficial for their wellbeing. When asked why peer support would be beneficial, $51.6 \%$ agreed that peer support would 
offer fewer restrictions, greater insight and understanding (49.5\%), be more accessible (43.9\%), give them greater choice than professional support $(40.4 \%)$, reduce workload for staff $(19.4 \%)$ and provide clearer and more relevant information (14.1\%). When asked if user-led support should happen more at university, half of participants (48.9\%) said that it should.

When asked what would stop them accessing peer support, most participants reported concerns around confidentiality (71.5\%), trust (60.6\%), anxiety $(55.3 \%)$, and whether peers would be skilled enough to support others $(48.9 \%)$ or that peers would not have the training staff have (36.2\%). Moreover, participants wanted to maintain boundaries between peers (30.3\%) and were concerned about the emotional impact of offering support (31.6\%) and stigma $(36 \%)$.

\section{Content analysis.}

Qualitative responses were provided by $223(59.3 \%)$ participants when asked why they thought peer support may or may not be beneficial. Content analysis categorised responses within broad categories of 'positive aspects' and 'negative aspects' of peer support (Figure 2). Within 'positive aspects', six categories were identified and agreed: mutual understanding, reducing loneliness and increasing friendship, meeting likeminded individuals, feeling more comfortable, sharing coping strategies and increasing accessibility to support. Within 'negative aspects', six categories were agreed: emotional barriers, lack of professionalism, stigma, concerns of exacerbating the problem, problems of confidentiality and trust and already having alternative social support. Inter-rater reliability for ten categories had almost perfect agreement (.80 - 1) and two categories had substantial agreement (.77 $.79)$.

[Insert Figure 2 here] 
The category within 'positive aspects' which had the most responses was mutual understanding $(n=62)$ - participants described benefits of meeting with people with a shared understanding of mental health difficulties: "Having someone who has been there before and come through the other side and can talk to you about how they did it and let you know that you are not alone". The second most reported category was around reducing loneliness and increasing friendship $(n=48)$ : "It would stop me feeling lonely if I had an avenue within which to make new friends who struggled with the same things that I do". The third most reported benefit was meeting likeminded individuals, who were similar to them in age and interests $(n=40)$; "You relate to those who are the same age as you therefore you may open up more and get more out of sessions".

For 'negative aspects', the category with most responses was emotional barriers $(n=26)$, such that participants described how they might find it difficult to share information with their peers: "I feel embarrassed about my mental health around my peers and might have an even harder time opening up". The second most reported category was lack of professionalism $(n=24)$, with participants expressing concerns over peers not being trained professionals: "Because students are not qualified to provide mental health support in times of crisis. It puts undue burden on students and places them in potential situations where they are unqualified to help and yet find that they must". The third most reported category was stigma $(n=9)$, with participants fearing that peers would judge them for using the service: "I don't feel that I should be talking to other students who may not know me and make judgements based on what I say to them. Could cause rumours". 


\section{Discussion}

The current study examined university students' experiences of NHS mental health services and potential benefits and challenges of peer support. Many participants reported that their experiences of NHS mental health services required improvement. Qualitative analysis identified issues including long waiting times, lack of availability of alternative treatments, a desire for more compassionate staff, concerns surrounding the effectiveness of current treatments, a lack of communication between services and a need for more follow-up care. Regarding peer support, most participants were unsure if they would access it. Most did agree that peer support would be beneficial for their wellbeing - qualitative data confirmed that many thought it would enable them to meet those with a mutual understanding of mental health difficulties at university. However, participants also noted concerns about peer support, believing it lacks professionalism, as well as concerns surrounding the potential shame or stigma from sharing personal information with peers. These findings suggest that peer support may be a useful service for some students, but appropriate training and clear guidelines on what peer support can and cannot offer should be disseminated to students.

\section{Experiences of NHS mental health services.}

Many participants reported a diagnosed mental health condition. Several suspected an undiagnosed condition, however most of these participants were not seeking a diagnosis. Over half had accessed support since starting university, most commonly through their GP. Overall, GP consultations were positively evaluated, however a lack of adequate time to discuss their condition was a frequently raised issue. Of the participants who had been in contact with NHS mental health services, most stated talking therapy to be partially helpful and desirable. However, over a third of participants who had received care from NHS mental health services in the past 12 months rated it as 'poor' or 'very poor'. Further, of those in 
contact with NHS mental health services, four in ten reported it as 'not helpful'. Qualitative responses provided deeper understanding of experiences with NHS mental health services: staff qualities and patient-centred communication and care were two identified positive aspects. However, it is notable that more responses were gathered when participants were asked what could be improved about their NHS care, implying there is room for improvement.

Several areas of improvement were identified, with waiting times frequently reported as an issue. This finding supports previous research on the experiences of NHS mental health services for adults within the general population (Docherty and Thornicroft 2015; Newman et al. 2015). Research has reported that long waiting times can result in poorer mental health outcomes, including more days in inpatient care and longer recovery times (Reichert and Jacobs 2018). This emphasises that reducing waiting times and facilitating timely access should be a priority for NHS mental health services. Long waiting times are likely exacerbated by a lack of funding, with treatment coverage (the proportion of those with a condition who received treatment) related to investment in mental health services (Docherty and Thornicroft 2015). This highlights a need for increased government funding for mental health services, which would benefit both students and the general population.

In the current study, patient-centred care was also an area of desired improvement, with only one in five participants reporting they 'definitely' had enough say in decisions about their care. First proposed by Byrne and Long (1976), patient-centred care enables patients to make informed decisions about their care in partnership with the clinician, including consideration of the needs and preferences of each patient when deciding on a treatment and care plan. Previous research suggests that applying this approach to mental health could have a positive impact on patient outcomes, including improvements in therapeutic relationships between 
clinicians and patients, adherence to treatments and improvements in symptoms (Doyle, Lennox and Bell 2013; Kornhaber et al. 2016). The current study highlights this is an aspect that also matters to students, and thus is a worthy focus for policy on student mental healthcare.

Participants also frequently reported issues with access - especially their opportunities to access alternative treatments, either alongside current services or as an alternative treatment. After their GP, the most used avenues of support were counselling services and mental health advisers within universities, suggesting that alternative forms of support are being sought and that students desire more help (Shaw 2014). Counselling services have shown significant improvements in students' levels of depression and anxiety (Royal College of Psychiatrists 2011), however students are not always aware of the services available to them (Kitzrow 2003). It is essential that communication increases between university mental health services, GPs and students, so that students are aware of all the possible options available to them.

Together, these findings suggest that there is an issue with students being able to access the support that they need, when they need it and with it being individualised to their specific needs. These unmet needs could exacerbate mental health issues, reduce the motivation to seek help and increase the risk of reoccurrence if left untreated (Zivin et al. 2009). The likelihood of students accessing support is related to several social and psychological barriers, such as self-stigma and past experiences (Cage et al. 2018). Even once support has been sought, the current study indicates that barriers continue to be faced, suggesting that current services do not adequately address the issue of student mental health. In the UK, there is arguably a long way to go before parity of esteem is reached for mental health care (Millard and Wessely 2014).

\section{Attitudes towards peer support.}


Peer support - although not an alternative to professional mental healthcare - could be beneficial if students are facing long waiting lists to access NHS support. In the current study, half of participants reported that user-led peer support should happen more at their university, with a third reporting that they would access peer support but most unsure if they would access it. This supports previous research demonstrating a desire for peer support services (Cunningham et al. 2017; Shaw 2014) although the number of unsure participants indicates that they may benefit from further information about peer support. The main reasons participants wanted peer support was to support recovery and meet new people. Qualitative responses added further insight into the perceived benefits of peer support, such as mutual understanding and meeting like-minded individuals. Supporting previous research, these findings suggest that students value being understood and relating to others (Naslund et al. 2014), and peer support could provide opportunities to normalise experiences (Dass and Gorman 1985).

Qualitative responses also indicated that reducing loneliness and increasing friendship were benefits. This benefit may be unique to peer support, and previous psychological research has highlighted the important role social belonging plays in mental wellbeing (e.g. McBeath et al. 2017). Indeed, Byrom (2018) found that students who attended six peer support group sessions experienced significant improvements in mental wellbeing. However, it is noteworthy that Byrom's (2018) study lacked a control group, making it harder to determine if these benefits are due to an increase in social support or specific benefits of a peer support service. More research is needed to evaluate the effectiveness of peer support on student mental health outcomes.

Despite peer support services being identified as a promising strategy for improving student mental health, potential limitations should be considered. In the current study, many 
participants had concerns over confidentiality, trust and anxiety. These potential limitations are regularly cited as main oppositions to peer support (Davidson et al. 2012; Shaw 2014). Discussing these concerns directly with students could be beneficial, such as by confirming that peer supporters would be trained appropriately and would respect confidentiality (Davidson et al. 2012). Additionally, qualitative responses further suggested that emotional barriers and stigma were common concerns. Emotional barriers are often raised as issues for young people when seeking help, with Rickwood et al. (2005) finding that young people who were less skilled at managing their emotions were less likely to seek help.

Together, these findings suggest that peer support could have emotional value, particularly helping to increase social support from others with shared experiences. However, emotional aspects also serve to limit the benefits of peer support, with fears over how others would perceive them if they used the service, and whether they could trust their peers. The effectiveness of peer support could therefore be limited by overarching stigmatizing attitudes towards mental health. Indeed, stigma has been identified as one of the most significant barriers for young people seeking help for mental health problems (Gulliver et al. 2010). Developing mental health literacy in students and staff may be an important tactic to ensure students are accessing the support they need and want.

\section{Limitations and future directions}

First, the generalisability of the current study is a significant limitation: most participants attended universities in South East England, suggesting that experiences of students in the current study may only be representative of NHS mental health services in this area. Further, most participants were female. Vidourek et al. (2014) reported that females are more likely seek social support for mental health problems, which may have contributed to the positive views on peer support in the current study. Despite this limited generalisability, the study's 
findings still have value in improving understanding of student experiences of NHS mental health services and perceptions of peer support services.

The study also assumes that intentional behaviour is a valid indicator of actual behaviour. Previous research has suggested that intentional behaviours for accessing mental health support does not always translate into actual behaviours (Li 2016). Therefore, students may like the concept of peer support and intend to access it, yet not use it. Further, the study assumes there no changes in the need for peer support services throughout a student's time at university. Previous research has suggested that certain time points at university such as the initial transition may be particularly difficult, increasing the need for additional support (Knoesen and Naude 2012). Thus, future longitudinal research could explore whether the need for peer support changes throughout university so that timely support can be offered.

Additionally, the study only gathered responses specifically on experiences of NHS mental health services, despite participants reporting that they had accessed other services such as mental health advisers and university counsellors. Previous research has reported that students value counselling services at university and these services are particularly beneficial when they have a formal link to the NHS (Quinn et al. 2009). Future research should consider student experiences of university mental health support services such as counselling, mental health advisers, disability services and mentors to develop a full understanding of mental health support for students.

Future research should also investigate the content of peer support services, to facilitate the provision of appropriate training and guidance. Davison et al. (2012) found that peer support staff reported that positive self-disclosure, role modelling and conditional regard were especially useful to increasing feelings of hope, control and a sense of belonging amongst 
users of the service. These findings suggest that exploring ways to effectively develop such skills in peer support staff is a worthy area of focus for future research.

Since stigma was noted as a barrier to receiving peer support both in the current study and elsewhere (Lucksted et al. 2011; Lawton-Smith 2013), online peer support services could be offered as an alternative option. This novel delivery method of peer support could offer advantages including increasing accessibility and allowing for anonymity. In a systematic review of online peer support, Ali et al. (2015) suggested that due to the anonymous nature of online support, as well as the common use of the internet to connect to others, online peer support may be an effective tool in reducing stigma and increasing help-seeking behaviour. In addition, online peer support could reduce waiting times for support, and allow students to access help outside of traditional opening times, such as weekday evenings, which was the preferred time to access peer support in the current study. Online peer support services may be particularly beneficial as lack of consistency in support has been noted as a problem for students due to short term dates (Brown 2018). This aspect is important as previous research has identified consistency in care and support as important factor in promoting positive mental health and recovery (Dixon, Holoshitz, and Nossel 2016).

\section{Conclusion}

The current study contributes to our understanding of student experiences of NHS mental health services and perceptions of peer support services. Several areas of improvement for mental health services were identified, including the need to reduce waiting times, improve access to alternative treatments and facilitate more patient-centred communication. Benefits of peer support services were also identified including the potential to normalise experiences and promote belonging, suggesting peer support could be a promising strategy for fostering better student mental health. Addressing the current problems with NHS mental health 
services and facilitating appropriate peer support services at university are a worthy focus for policy development for student mental health support. 


\section{References}

Ali, K., L. Farrer, A. Gulliver, and K. M. Griffiths. 2015. “Online Peer-to-Peer Support for Young People with Mental Health Problems: A Systematic Review”. Journal of Medical Internet Research Mental Health 2 (2): e19. doi: 10.2196/mental.4418.

Bask, M. 2015. "Externalising and internalising problem behaviour among Swedish adolescent boys and girls.” International Journal of Social Welfare 24 (2): 182-192. doi: 10.1111/ijsw.12106.

Biasi, Valeria, Nazarena Patrizi, Mauro Mosca, and Conny De Vincenzo. 2017. "The Effectiveness of University Counselling for Improving Academic Outcomes and Well-Being.” British Journal of Guidance \& Counselling 45 (3): 248-257. doi: 10.1080/03069885.2016.1263826.

Brown, P. 2018. The invisible problem? Improving students' mental health. Higher Education Policy Institute. Accessed 31 July 2018. https://www.hepi.ac.uk/wpcontent/uploads/2016/09/STRICTLY-EMBARGOED-UNTIL-22-SEPT-Hepi-Report$\underline{\text { 88-FINAL.pdf }}$

Byrne, P. S., and B.E L. Long. 1976. Doctors Talking to Patients. London: HSMO.

Byrom, N. 2018. “An Evaluation of a Peer Support Intervention for Student Mental Health.” Journal of Mental Health 27 (3): 240-246. doi: 10.1080/09638237.2018.1437605.

Cage, E., M. Stock, A. Sharpington, E. Pitman, and R. Batchelor. 2018. "Barriers to accessing support for mental health issues at university." Studies in Higher Education: 1-13. doi: 10.1080/03075079.2018.1544237. 
Commission for Healthcare Audit and Inspection. 2008. Service user questionnaire. Accessed 31 July 2018.

http://www.nhssurveys.org/Filestore//documents/MH08 Scored Questionnaire.pdf

Cornwall, Andrea, and Rachel Jewkes. 1995. "What is Participatory Research?” Social Science \& Medicine 41 (12): 1667-1676. doi: 10.1016/0277-9536(95)00127-s.

Cruwys, Tegan., S. Alexander Haslam, Genevieve A. Dingle, Jolanda Jetten, Matthew J. Hornsey, E. M. Desdemona Chong, and Tian P. S. Oei. 2014. "Feeling connected again: Interventions that increase social identification reduce depression symptoms in community and clinical settings. Journal of Affective Disorders 159: 139-146. doi: 10.1016/j.jad.2014.02.019.

Cunningham, C. E., R. B. Zipursky, B. K. Christensen, P. J. Bieling, V. Madsen, H. Rimas, S. Mielko, et al. 2017. "Modelling the mental health service utilization decisions of university undergraduates: A discrete choice conjoint experiment." Journal of American College Health 65 (6): 389-399. doi: 10.1080/07448481.2017.1322090.

Dass, Ram, and Paul Gorman. 1985. How Can I Help? Stories and Reflections on Service. New York: Alfred A. Knopf.

Davison, Larry, Chyrell Bellamy, Kimberly Guy, and Rebecca Miller. 2012. "Peer Support Among Persons with Severe Mental Illnesses: A Review of Evidence and Experience." Mental Health Policy Paper 11 (2): 123-128. https://www.ncbi.nlm.nih.gov/pmc/articles/PMC3363389/.

Dixon, L. B., Y. Holoshitz, and I. Nossel. 2016. "Treatment engagement of individuals experiencing mental illness: review and update." World Psychiatry 15 (1): 13-20. doi: 10.1002/wps.20306. 
Docherty, Mary, and Graham Thornicroft. 2015. "Specialist mental health services in England in 2014: overview of funding, access and levels and care." International Journal of Mental Health Systems 9 (1). doi: 10.1186/s13033-015-0023-9.

Doyle, Cathal, Laura Lennox, and Derek Bell. 2013. "A Systematic Review of Evidence on the Links Between Patient Experience and Clinical Safety and Effectiveness.” British Medical Journal Open 3 (1): e001570. doi: 10.1136/bmjopen-2012-001570.

Eisenberg, Daniel, Ezra Golberstein, and Justin B. Hunt. 2009. "Mental Health and Academic Success in College.” The B.E. Journal of Economic Analysis \& Policy 9 (1): 1-37. https://ideas.repec.org/a/bpj/bejeap/v9y2009i1n40.html.

Gulliver, A., L. Farrer, K. Bennett, K. Ali, A. Hellsing, N. Katruss, and K. M. 2018. "University staff experiences of students with mental health problems and their perceptions of staff training needs.” Journal of Mental Health 27 (3): 247-256.doi: 10.1080/09638237.2018.1466042.

Gulliver, A., K. M. Griffiths, and H. Christensen. 2010. "Perceived barriers and facilitators to mental health help-seeking in young people: a systematic review." BMC Psychiatry 10 (1): 113. doi: 10.1186/1471-244x-10-113.

Higher Education Statistics Authority. 2018. Higher Education Student Statistics: UK, 201617 - student numbers and characteristics. HESA. Accessed 31 July 2018. https://www.hesa.ac.uk/news/11-01-2018/sfr247-highereducation-student$\underline{\text { statistics/numbers }}$

Hsieh, H. F., and S. E. Shannon. 2005. "Three approaches to qualitative content analysis." Qualitative Health Research 15 (9): 1277-1288. doi: 10.1177/1049732305276687. 
Kendall, Tim, Mike J. Crawford, Clare Taylor, Craig Whittington, and Diana Rose. 2012. "Improving the experience of care for adults using NHS mental health services: summary of NICE guidance." British Medical Journal 344: e1089. doi:10.1136/bmj.e1089.

Kitzrow, Martha Anne. 2003. “The Mental Health Needs of Today’s College Students: Challenges and Recommendations.” Journal of Student Affairs Research and Practice 41 (1): 167-181. doi:10.2202/1949-6605.1310.

Knoesen, R., and L. Naude. 2017. "Experiences of flourishing and languishing during the first year at university." Journal of Mental Health 27 (3): 269-278. doi: 10.1080/09638237.2017.1370635.

Kornhaber, Rachel, Kenneth Walsh, Jed Duff, and Kim Walker. 2016. "Enhancing adult therapeutic interpersonal relationships in acute health care setting: an integrative review." Journal of Multidisciplinary Healthcare 9: 537-546. doi: 10.2147/JMDH.S116957.

Landis, Richard D., and Gary G. Koch. 1977. "The Measurement of Observer Agreement for Categorical Data.” Biometrics 33 (1): 159-174. doi: 10.2307/2529310.

Lawton-Smith, Simon. 2013. "Peer support in mental health: where are we today?" The Journal of Mental Health Training, Education and Practice 8 (3): 152-158. doi:10.1108/jmhtep-03-2013-0009.

Li, Wenjing. 2016. 'University students' mental health help-seeking: intention and service use.” PhD diss., The University of Adelaide. 
Lucksted, A., A. Drapalski, C. Calmes, C. Forbes, B. DeForge, and J. Boyd. 2011. “Ending self-stigma: pilot evaluation of a new intervention to reduce internalized stigma among people with mental illnesses." Psychiatric Rehabilitation Journal 35 (1): 5154. doi: 10.2975/35.1.2011.51.54.

McBeath, Margaret, Maureen T. B. Drysdale, and Nicholas Bohn. 2017. "Work-integrated learning and the importance of peer support and sense of belonging." Education + Training 60 (1): 39-53. doi:10.1108/et-05-2017-0070.

Millard, C., and S. Wessely. 2014. "Parity of esteem between mental and physical health." BMJ, 349: g6821. doi:10.1136/bmj.g6821.

Mowbray, C. R., D. Megivern, J. M. Mandiberg, S. Strauss, C. H. Stein, K. Collins, S. Kopels, C. Curlin, and R. Lett. 2006. "Campus mental health services: Recommendations for change.” American Journal of Orthopsychiatry 76 (2): 226277. doi: 10.1037/0002-9432.76.2.226.

Naslund, John A., Stuart W. Grande, Kelly A. Aschbrenner, and Glyn Elwyn. 2014. 'Naturally Occurring Peer Support Through Social Media: The Experiences of Individuals with Severe Mental Illness Using YouTube.” PLoS ONE 9 (10): e110171.doi:10.1371/journal.pone.0110171.

Newman, D., P. O’Reily, S. H. Lee, and C. Kennedy. 2015. 'Mental health services users' experiences of mental health care: an integrative literature review." Journal of Psychiatric and Mental Health Nursing 22 (3): 171-182. doi: 10.1111/jpm.12202.

NHS Digital. 2018. Psychological therapies: Annual report on the use of IAPT services England, further analyses on 2016-17. Accessed 31 July 2018. https://files.digital.nhs.uk/publication/s/n/psyc-ther-ann-rep-2016-17 add.pdf 
NUS-USI. 2017. Student Wellbeing Research report.Accessed 31 July 2018.

https://nusdigital.s3-eu-west-

1.amazonaws.com/document/documents/33436/59301ace47d6320274509b83e1bea53

e/NUSUSI_Student_Wellbeing_Research_Report.pdf

Quinn, Neil, Alistair Wilson, Gillian MacIntyre, and Teresa Tinklin. 2009. “People look at you differently': Students' experience of mental health support within Higher Education.” British Journal of Guidance \& Counselling 37 (4): 405-418. doi:10.1080/03069880903161385.

Reichert, Anika, and Rowena, Jacobs. 2018. "The impact of waiting time on patient outcomes: Evidence from early intervention in psychosis services in England." Health Economics 27 (11): 1772-1787. doi: 10.1002/hec.3800.

Rickwood, D., Frank P. Deane, Coralie J. Wilson, and Joseph V. Ciarrochi. 2005. "Young people's help-seeking for mental health problems." Australian e-Journal for the Advancement of Mental Health 4 (3): 218-251. doi:10.5172/jamh.4.3.218.

Rosenthal, B., and W. C. Wilson. 2008. "Mental health services: Use and disparity among diverse college students. ” Journal of American College Health 57 (1): 61-68. doi: 10.3200/JACH.57.1.61-68.

Royal College of Psychiatrists. 2011. Mental Health of Students in Higher Education (College Report CR166). Accessed 31 July 2018. https://www.rcpsych.ac.uk/files/pdfversion/CR166.pdf

Sani, Fabio, Marina Herrera, Juliet. R. H. Wakefield, Olga Boroch, and Csilla Gulyas. 2012. "Comparing social contact and group identification as predictors of mental health." 
British Journal of Social Psychology 51 (4): 781-790. doi:10.1111/j.20448309.2012.02101.x.

Shaw, Claire. 2014. Peer Support in Secure Services. Together: For mental wellbeing. Accessed 31 July 2018. http://www.together-uk.org/wpcontent/uploads/downloads/2014/11/Peer-Support-in-Secure-Settings-Final-Report-4$\underline{\text { Nov-14.pdf }}$

Thorley, Craig. 2017. Not By Degrees: Improving student mental health in the UK's Universities, IPPR. Accessed 31 July 2018. http://www.ippr.org/research/publications/not-by-degrees

Vidourek, Rebecca A., Keith A. King, Laura A. Nabors, and Ashley L. Merianos. 2014. "Students' benefits and barriers to mental health help-seeking." Health Psychology and Behavioral Medicine 2 (1): 1009-1022. doi:10.1080/21642850.2014.963586.

Wong, Kah P., Gregory Bonn, Cai L. Tam, and Chee P. Wong. 2018. "Preferences for Online and/or Face-to-Face Counselling among University Students in Malaysia.” Frontiers in Psychology 9 (64). doi:10.3389/fpsyg.2018.00064.

Zivin, K., D. Eisenberg, S. E. Gollust, and E. Golberstein. 2009. "Persistence of mental health problems and needs in a college student population." Journal of Affective Disorders 117 (3): 180-185. doi:10.1016/j.jad.2009.01.001. 
Table 1. Diagnostic information on mental health conditions in the sample.

\begin{tabular}{llll}
\hline Condition & $\begin{array}{l}\text { Percentage } \\
\text { reporting } \\
\text { diagnosis }\end{array}$ & $\begin{array}{l}\text { Percentage } \\
\text { diagnosed during } \\
\text { university }\end{array}$ & $\begin{array}{l}\text { Median age of } \\
\text { diagnosis }\end{array}$ \\
\hline Anxiety disorder & $30.3 \%$ & $33.3 \%$ & 17 \\
Mood disorder & $22.3 \%$ & $27.3 \%$ & 16 \\
Eating disorder & $8 \%$ & $23.3 \%$ & 15 \\
Obsessive Compulsive Disorder & $5.9 \%$ & $36.4 \%$ & 17 \\
Personality disorder & $3.7 \%$ & $28.5 \%$ & 19 \\
Post-Traumatic Stress Disorder & $3.7 \%$ & $50 \%$ & 19 \\
Psychotic Disorder & $1.6 \%$ & $33.3 \%$ & 17.5 \\
Other & $1.6 \%$ & $25 \%$ & 16 \\
Addiction disorder & $.8 \%$ & $33.3 \%$ & 17 \\
\hline Note. Participants & & \\
\hline
\end{tabular}

Note. Participants could report more than one condition. 
Table 2. Support types that had been accessed for mental health during university.

\begin{tabular}{ll}
\hline Support service & N (\% of sample) \\
\hline I have not accessed any services for my mental health & $163(43.4 \%)$ \\
General Practitioner & $152(40.4 \%)$ \\
Counselling at university & $119(31.6 \%)$ \\
Mental health adviser at university & $77(20.5 \%)$ \\
Counselling outside of university & $64(17 \%)$ \\
Disability services at university & $62(16.5 \%)$ \\
Psychiatrist & $40(10.6 \%)$ \\
Clinical Psychologist & $31(8.2 \%)$ \\
Community Psychiatric Nurse & $14(3.7 \%)$ \\
Other & $10(2.7 \%)$ \\
\hline
\end{tabular}

Note. Participants could select more than one support service. 
Table 3: Participants views on the qualities of their GP during their last meeting to discuss their mental health.

\begin{tabular}{llll}
\hline GP quality & $\begin{array}{l}\text { Yes, } \\
\text { definitely }\end{array}$ & $\begin{array}{l}\text { Yes, to some } \\
\text { extent }\end{array}$ & No \\
\hline Listened to & $52(35.6 \%)$ & $65(44.5 \%)$ & $29(19.9 \%)$ \\
Trusted/had confidence in them & $57(39 \%)$ & $58(39.7 \%)$ & $31(21.2 \%)$ \\
Treated with dignity and respect & $85(58.2 \%)$ & $41(28.1 \%)$ & $20(13.7 \%)$ \\
Adequate time to discuss condition and treatment & $39(26.7 \%)$ & $40(27.4 \%)$ & $67(45.9 \%)$ \\
\hline
\end{tabular}


Table 4. Ways students support each other at participants' universities and what types of support are needed. Note: Participants could select more than one support service.

\begin{tabular}{lll}
\hline Type of Support & $\begin{array}{l}\text { Ways that students } \\
\text { support each other at } \\
\text { university: } \\
\text { n(\% of sample) }\end{array}$ & $\begin{array}{l}\text { What types of support } \\
\text { are needed at } \\
\text { university: }\end{array}$ \\
& $\begin{array}{l}\boldsymbol{n} \text { (\% of sample) } \\
\text { 1:1 support }\end{array}$ & $213(56.6 \%)$ \\
Support in a crisis & $78(20.7 \%)$ & $205(54.5 \%)$ \\
Telephone/Online support & $154(41 \%)$ & $189(50.3 \%)$ \\
Drop in sessions & $132(35.1 \%)$ & $181(48.1 \%)$ \\
Relaxation \& Mindfulness & $156(41.5 \%)$ & $180(47.9 \%)$ \\
Condition-based groups & $53(14.1 \%)$ & $177(47.1 \%)$ \\
Young adult (18-25) support groups & $29(7.7 \%)$ & $175(46.5 \%)$ \\
Activity groups & $200(53.2 \%)$ & $137(36.4 \%)$ \\
Develop new life skills & $48(12.8 \%)$ & $131(34.8 \%)$ \\
Help to link with wider community activities & $52(13.8 \%)$ & $121(32.2 \%)$ \\
Recovery Star & $6(1.6 \%)$ & $120(31.9 \%)$ \\
Recovery stories & $24(6.4 \%)$ & $117(31.1 \%)$ \\
Support groups for new mothers & $5(1.3 \%)$ & $92(24.5 \%)$ \\
\hline & & \\
\hline
\end{tabular}






Figure 1. Categories and subcategories from the content analysis on qualitative responses on improvements to NHS MH services 


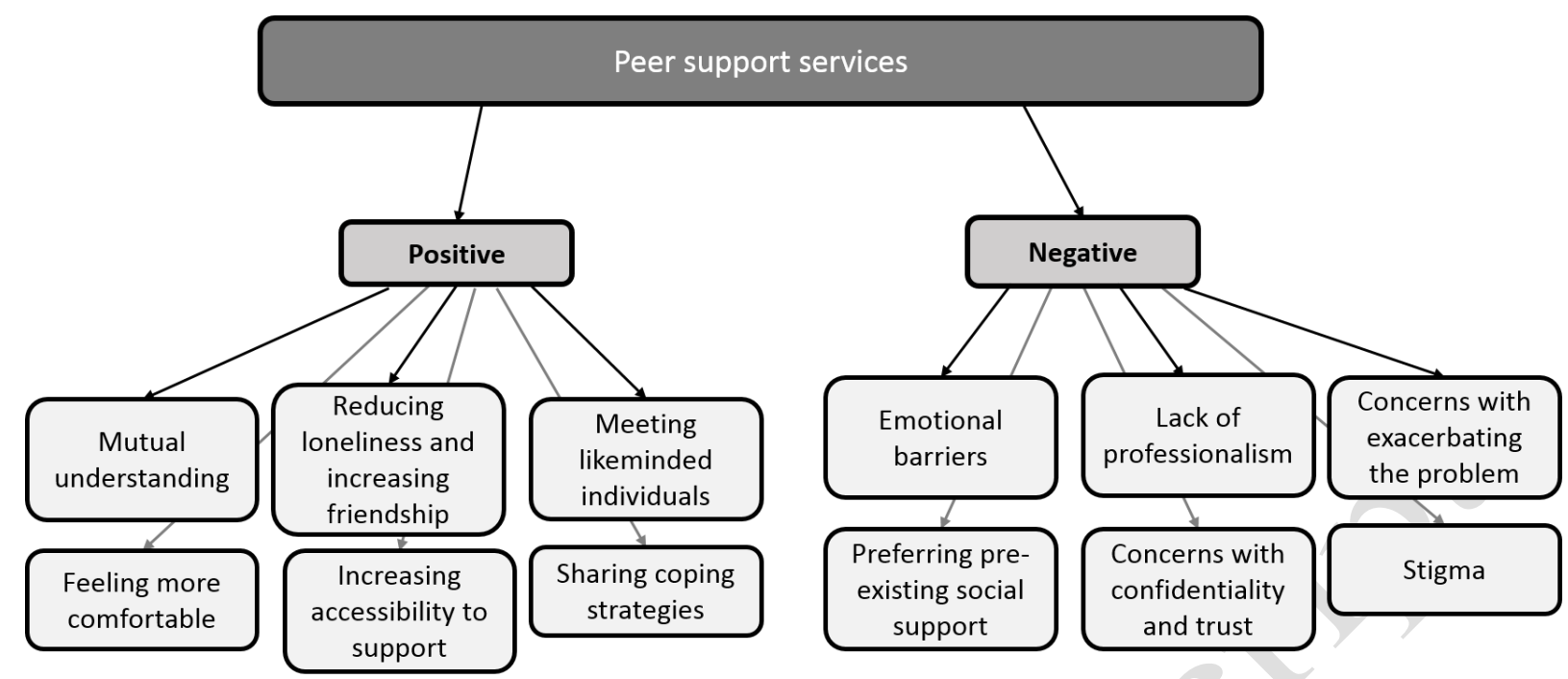

Figure 2. Categories from the content analysis on qualitative responses on peer support services 